Luiz Fernando de Moura $\cdot$ Roger E. Hernández

\title{
Effects of abrasive mineral, grit size and feed speed on the quality of sanded surfaces of sugar maple wood
}

Published online: 25 May 2006

(C) Springer-Verlag 2006

\section{Wood Sci Technol (2006) DOI 10.1007/s00226-006-0070-0}

Due to a processing error, the presentation of Table 1 was incorrect [in the HTML version of this article]. The corrected table is given below.

Table 1 Average roughness average $\left(R_{\mathrm{a}}\right)$ of sugar maple sanded surfaces measured along and across the grain for three sanding programs, four feed speeds and two abrasive minerals

\begin{tabular}{|c|c|c|c|c|c|}
\hline \multirow{2}{*}{$\begin{array}{l}\text { Sanding } \\
\text { program } \\
\text { (grit) }\end{array}$} & \multirow{2}{*}{$\begin{array}{l}\text { Feed } \\
\text { speed } \\
(\mathrm{m} / \mathrm{min})\end{array}$} & \multicolumn{2}{|c|}{ Aluminum oxide ${ }^{a}$} & \multicolumn{2}{|c|}{ Silicon carbide $^{\mathrm{a}}$} \\
\hline & & $R_{\mathrm{a} \|}^{\mathrm{b}}(\mu \mathrm{m})$ & $R_{\mathrm{a} \perp}^{\mathrm{c}}(\mu \mathrm{m})$ & $R_{\mathrm{a} \|}(\mu \mathrm{m})$ & $R_{\mathrm{a} \perp}(\mu \mathrm{m})$ \\
\hline 100 & $\begin{array}{l}8 \\
10 \\
12 \\
14\end{array}$ & $\begin{array}{l}2.9(0.2)^{\mathrm{d}} \mathrm{A}^{\mathrm{e}} \mathrm{b} \\
2.9(0.1) \mathrm{A} \mathrm{b} \\
3.1(0.2) \mathrm{A} \mathrm{b} \\
3.4(0.2) \mathrm{A} \mathrm{b}\end{array}$ & $\begin{array}{l}5.5(0.1) \mathrm{A} \mathrm{b} \\
5.9(0.2) \mathrm{AB} \mathrm{b} \\
5.9(0.3) \mathrm{AB} \mathrm{b} \\
6.4(0.2) \mathrm{B} \mathrm{b}\end{array}$ & $\begin{array}{l}2.9(0.2) \mathrm{A} \mathrm{b} \\
2.9(0.2) \mathrm{A} \mathrm{b} \\
3.0(0.2) \mathrm{A} \mathrm{b} \\
3.5(0.2) \mathrm{A} \mathrm{b}\end{array}$ & $\begin{array}{l}5.4(0.2) \mathrm{A} \mathrm{b} \\
5.1(0.2) \mathrm{A} \mathrm{b} \\
5.5(0.2) \mathrm{A} \mathrm{b} \\
5.8(0.3) \mathrm{A} \mathrm{b}\end{array}$ \\
\hline $\begin{array}{l}100 \\
120\end{array}$ & $\begin{array}{l}8 \\
10 \\
12 \\
14\end{array}$ & $\begin{array}{l}2.2(0.1) \mathrm{A} \mathrm{a} \\
2.4(0.1) \mathrm{A} \mathrm{a} \\
2.4(0.2) \mathrm{A} \mathrm{a} \\
2.5(0.1) \mathrm{A} \mathrm{a}\end{array}$ & $\begin{array}{l}3.5(0.1) \mathrm{A} \mathrm{a} \\
4.0(0.2) \mathrm{AB} \mathrm{a} \\
4.2(0.2) \mathrm{AB} \mathrm{a} \\
4.3(0.2) \mathrm{B} \mathrm{a}\end{array}$ & $\begin{array}{l}2.1(0.1) \mathrm{A} \mathrm{a} \\
2.2(0.1) \mathrm{A} \mathrm{a} \\
2.1(0.1) \mathrm{A} \mathrm{a} \\
2.4(0.1) \mathrm{A} \mathrm{a}\end{array}$ & $\begin{array}{l}3.3(0.1) \mathrm{A} \mathrm{a} \\
3.8(0.2) \mathrm{AB} \mathrm{a} \\
4.0(0.1) \mathrm{B} \mathrm{a} \\
3.9(0.2) \mathrm{B} \mathrm{a}\end{array}$ \\
\hline $\begin{array}{l}100 \\
120 \\
150\end{array}$ & $\begin{array}{l}8 \\
10 \\
12 \\
14\end{array}$ & $\begin{array}{l}1.9(0.1) \mathrm{A} \mathrm{a} \\
2.4(0.1) \mathrm{B} \mathrm{a} \\
2.1(0.1) \mathrm{AB} \mathrm{a} \\
2.3(0.2) \mathrm{AB} \mathrm{a}\end{array}$ & $\begin{array}{l}3.8(0.1) \mathrm{A} \mathrm{a} \\
4.2(0.2) \mathrm{AB} \mathrm{a} \\
4.5(0.1) \mathrm{B} \mathrm{a} \\
4.5(0.2) \mathrm{B} \mathrm{a}\end{array}$ & $\begin{array}{l}2.0(0.1) \mathrm{A} \mathrm{a} \\
2.0(0.1) \mathrm{A} \mathrm{a} \\
2.1(0.1) \mathrm{A} \mathrm{a} \\
2.2(0.1) \mathrm{A} \mathrm{a}\end{array}$ & $\begin{array}{l}3.5(0.2) \mathrm{A} \mathrm{a} \\
3.9(0.2) \mathrm{A} \mathrm{a} \\
3.9(0.1) \mathrm{A} \mathrm{a} \\
3.6(0.1) \mathrm{A} \mathrm{a}\end{array}$ \\
\hline
\end{tabular}

\footnotetext{
${ }^{\mathrm{a}}$ Means of 20 replicates

${ }^{\mathrm{b}}$ Roughness average measured along the grain

${ }^{\mathrm{c}}$ Roughness average measured across the grain

${ }^{\mathrm{d}}$ Standard error of the mean in parentheses

${ }^{\mathrm{e}}$ Means within a column followed by the same letter are not significantly different at the $5 \%$ probability level (Tukey's test). Uppercase letters are for feed speed comparison, for each sanding program separately. Lowercase letters are for sanding program comparison, for each feed speed separately
}

The online version of the original article can be found at http://dx.doi.org/10.1007/s00226-006-0070-0

L. F. de Moura · R. E. Hernández ( $₫)$

Département des Sciences du Bois et de la Forêt, Université Laval,

G1K 7P4, Quebec, Canada

E-mail: Roger.Hernandez@sbf.ulaval.ca 